\title{
The role of basic plasmas studies in the quest for fusion power
}

\author{
Efforts to demonstrate the feasibility of fusion power can benefit from studies of fundamental questions in plasma \\ physics carried out in simplified devices.
}

Ambrogio Fasoli, Ivo Furno and Paolo Ricci

O $\mathrm{n}$ the road to fusion power as a viable energy source, the Joint European Torus tokamak holds the record for the highest energy gain achieved with $16 \mathrm{MW}$ of fusion power generated from $24 \mathrm{MW}$ of input heating power. The next-generation tokamak known as the International Thermonuclear Reactor (ITER) aims for a 10-fold net energy gain of 500 MW from 50 MW heating power. The success of the ITER experiment is hence crucial for future fusion reactors ${ }^{1}$. In parallel to the development of the ITER tokamak, the next step towards the first commercial power plants ${ }^{2}$ has to be conceived. With both these goals in mind, the fusion community needs to find a balance between focusing on reactor technology and on refining the physical understanding of fusion plasmas. A substantial contribution in this direction can be achieved by conducting experiments in simplified plasma devices, and transferring the results to tokamaks or stellarators.

A better knowledge of the underlying physical processes allows technology problems to be better defined, which can therefore be treated more easily. On the other hand, technology hurdles pragmatically dictate the problems that must be tackled most urgently - possibly via an understanding of their fundamental physical aspects. As previous generations of tokamaks have shown some of these phenomena might already be apparent in their entirety, but not necessarily in their full-fledged consequences. Examples include the exhaust of plasma particles and heat from the confinement region to the pumped edge of the devices, and the interaction between the plasma and the surrounding material surfaces ${ }^{3}$. Other phenomena are intrinsic to regimes and conditions that are beyond what we can observe today, such as those associated with the strong self-heating of the plasma by the by-products of fusion reactions ${ }^{4}$.

By isolating a single aspect of an observed phenomenon and by reproducing it in a simplified set-up, it can be measured in

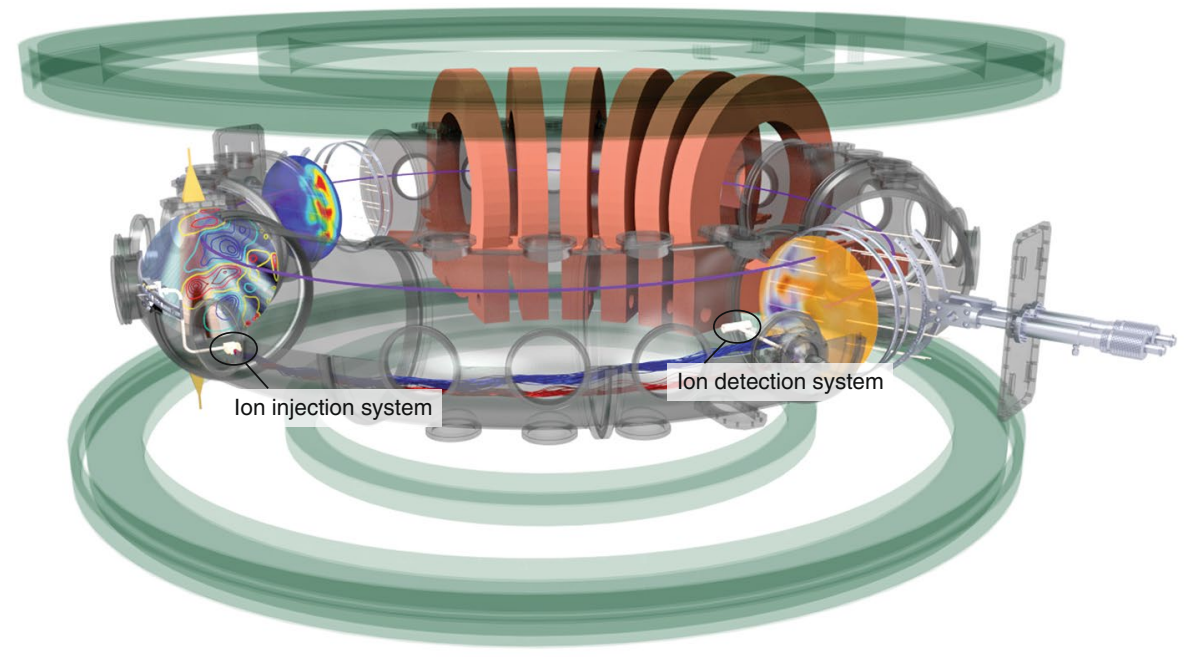

Fig. 1 | The TORPEX device. The suprathermal ion injection and detection systems are labelled. The blue and red lines indicate examples of suprathermal ion trajectories for two different injection energies, while the violet line indicates the magnetic field. Cross-sections with the representation of plasma turbulence are also shown on both sides, with blue and orange background, as calculated using the numerical GBS code simulating plasma dynamics at the edge of fusion devices ${ }^{30}$. The arrays of electrostatic probes mounted on metallic rings and used to reconstruct the blob dynamics are shown on the right. The arrangement specifically developed for the measurement of the scattering of millimetre waves by plasma turbulence and blobs is shown on the left. In particular, one can see the horn used to inject the waves vertically, and the visualization of the wave propagation through the density blobs, indicated by the contours in front of the horn.

detail. Then, the interplay between the individual physical elements that contribute to this phenomenon can be studied, and one can determine which of these elements are susceptible to external control - even in the strongly self-organized system in reactor-grade plasmas. Together with basic experimentation, numerical simulations are developed on the basis of first-principles or semi-phenomenological approaches to address the open questions in fusion research. These simulations have to be carefully validated. One of the main challenges is to identify in which circumstances these validation platforms can be much simpler than the high-performance fusion devices themselves and to establish criteria on when such tests can be considered conclusive in view of predicting much larger experimental realizations.

Several experimental devices built and operated with relatively modest investments have been developed worldwide to solve issues related to basic plasma physics, informing not only fusion research, but impacting often what we know of plasmas in space and astrophysical environments $^{5-13}$. These basic plasma facilities have contributed to the explanation of a number of physics problems, as well as to the education of students and young scientists in an environment that is more prone to training than of major fusion infrastructures.

One of those devices is TORPEX, shown in Fig. 1, operated at the EPFL Swiss Plasma 


\section{Box 1 | The TORPEX device}

In TORPEX, plasmas are created by heating different gases with microwaves at $2.45 \mathrm{GHz}$ and are characterized by low electron densities of $10^{16}-10^{17} \mathrm{~m}^{-3}$ and temperatures of $T_{\mathrm{e}} \sim 5-20 \mathrm{eV}$. In the simple magnetized torus (SMT) configuration, plasmas are confined by a toroidal magnetic field up to $B_{\mathrm{T}}=0.1 \mathrm{~T}$, and a smaller vertical component $B_{z} \leq 50 \mathrm{mT}$. The SMT incorporates the main ingredients for drift and interchange instabilities and turbulence, namely pressure gradients, magnetic field line curvature and open field lines. In addition to SMT configurations, an internal toroidal conductor system allows creating twisted field lines with rotational transform, thus creating a region of closed field lines and magnetic surfaces as prevalent in tokamak configurations. The relatively low temperatures and densities of TORPEX plasmas allow using internal probes for a full reconstruction of plasma profiles and turbulent structures across the entire device. TORPEX possesses an extensive set of diagnostics, comprising approximately 200 probes, together with a fast optical imaging system as well as a specialized set-up to inject and detect suprathermal ions.

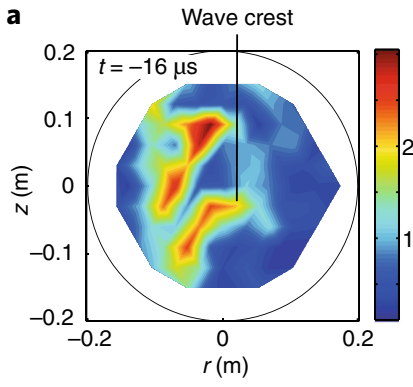

d

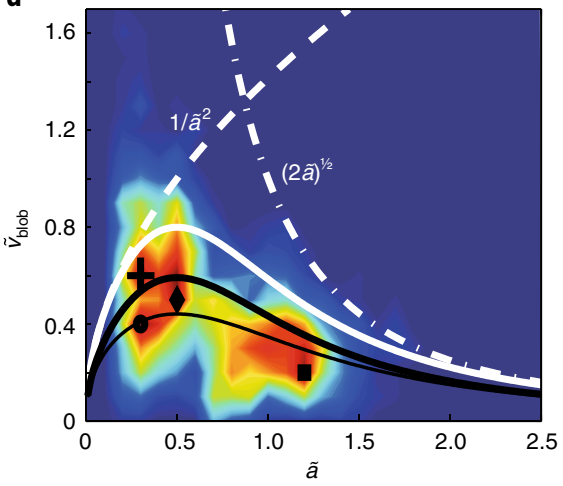

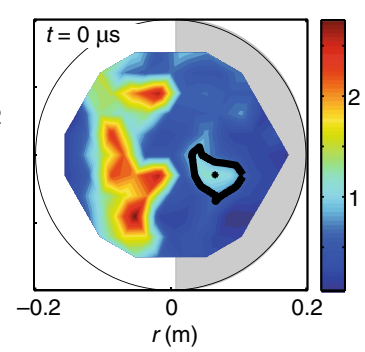

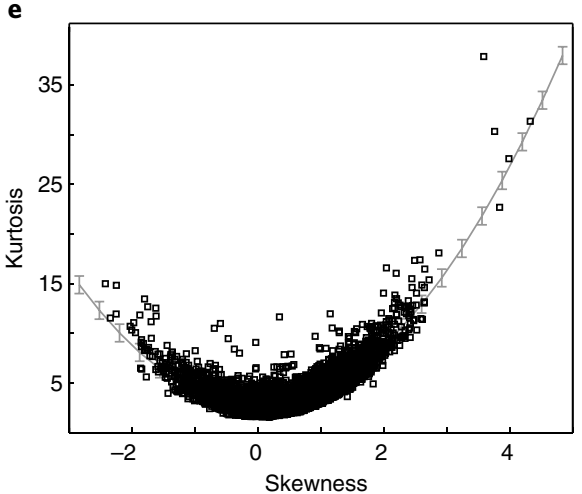

Fig. 2 | Blob formation mechanism. a-c, Visualization of the mechanism for blob formation in the interchange-dominated turbulence of TORPEX. The panels show the detachment of the density blob from the shearing of the interchange wave crest over the TORPEX plasma cross-section ${ }^{15} . z$ and $r$ indicate the vertical and radial directions of the plasma cross-section and $t$ the time ( $t=0$ being the instant at which a blob is detected on a reference probe). The colour bars represent plasma density in arbitrary units. d, The scaling of the blob velocity $\left(\tilde{v}_{\text {blob }}\right)$ with its size $(\tilde{a})^{18}$, both expressed in normalized units. The colours indicate the joint probability of velocity versus size, and the different symbols show the peaks of the distributions for the different gases used in the experiment $\left(\mathrm{H}_{2}\right.$, square; He, diamond; $\mathrm{Ne}$, cross; $\mathrm{Ar}$, circle). The solid black and white curves indicate the analytical scaling derived in ref. ${ }^{18}$, evaluated for different blob amplitudes and ion-neutral collision frequencies. e, The universal statistical features of the turbulence observed in TORPEX, in terms of a general relation between the third- and fourth-order moments of the probability density distribution ${ }^{21}$. The line indicates a least-square fit by a quadratic polynomial, and the error bars correspond to the $95 \%$ confidence bound in the fit. Similar curves are reconstructed from measurements of turbulent fluctuations in many basic and edge tokamak plasmas. Figure reproduced from: a-c, ref. ${ }^{15}$, Cambridge University Press; d, ref. ${ }^{18}$, American Physical Society; e, ref. ${ }^{21}$, American Physical Society.

Center (Box 1). TORPEX aims to contribute to the basic understanding of fundamental plasma physics problems of interest for burning fusion plasmas - by direct discoveries and by validating the relevant numerical models and codes ${ }^{14,15}$. The focus of research carried out with TORPEX lies on turbulence, which is a ubiquitous feature of magnetized plasmas. Generated by the nonlinear development of plasma instabilities, turbulence plays a crucial role in the transport of energy and particles both in the core and at the edge of the plasma. Recent measurements and modelling have recognized that, within the turbulent evolution of plasma instabilities, quasicoherent, mesoscale structures form and live over relatively long timescales. These turbulent structures strongly influence transport of thermal and non-thermal plasma components, leading to unforeseen phenomena such as self-regulation of flows, intermittency and scattering of externally launched waves. The investigations of these structures in the TORPEX device elucidate effects that may be of relevance for burning plasmas in ITER, and eventually, commercial fusion power plants.

The transport of particles, energy and momentum across the magnetic field is often characterized by the presence of coherent filamentary plasma structures. The temperature and density in these so-called blobs or filaments, which extend much further in the direction of the magnetic field than perpendicular to it, are enhanced with respect to the background plasma. In particular, blobs largely contribute to the transport across the plasma edge, and hence can possibly lead to serious wall erosion or to the release of impurities from the wall material.

When the first blobs in plasmas were observed $^{16}$, it was unclear how they were generated in the course of the nonlinear evolution of plasma instabilities and turbulence. In the TORPEX device, the mechanism of blob formation in scenarios dominated by ideal interchange turbulence, reminiscent of Rayleigh-Taylor instabilities in fluids, was identified. Blobs form from radially extending positive crests of the interchange waves that are sheared off by the motion of the particles resulting from the presence of a magnetic and electric field, known as the $\mathbf{E} \times \mathbf{B} \mathrm{drift}^{17}$. Once formed, the blob's motion is a result of the gradient and curvature of the magnetic field, which cause blob polarization and a corresponding $\mathbf{E} \times \mathbf{B} \mathrm{drift}^{18}$. The same mechanisms are at play both in a simple helical magnetic field configuration and in the presence of a magnetic X-point ${ }^{19}$.

As an ultimate demonstration that the fundamental mechanisms regulating the 

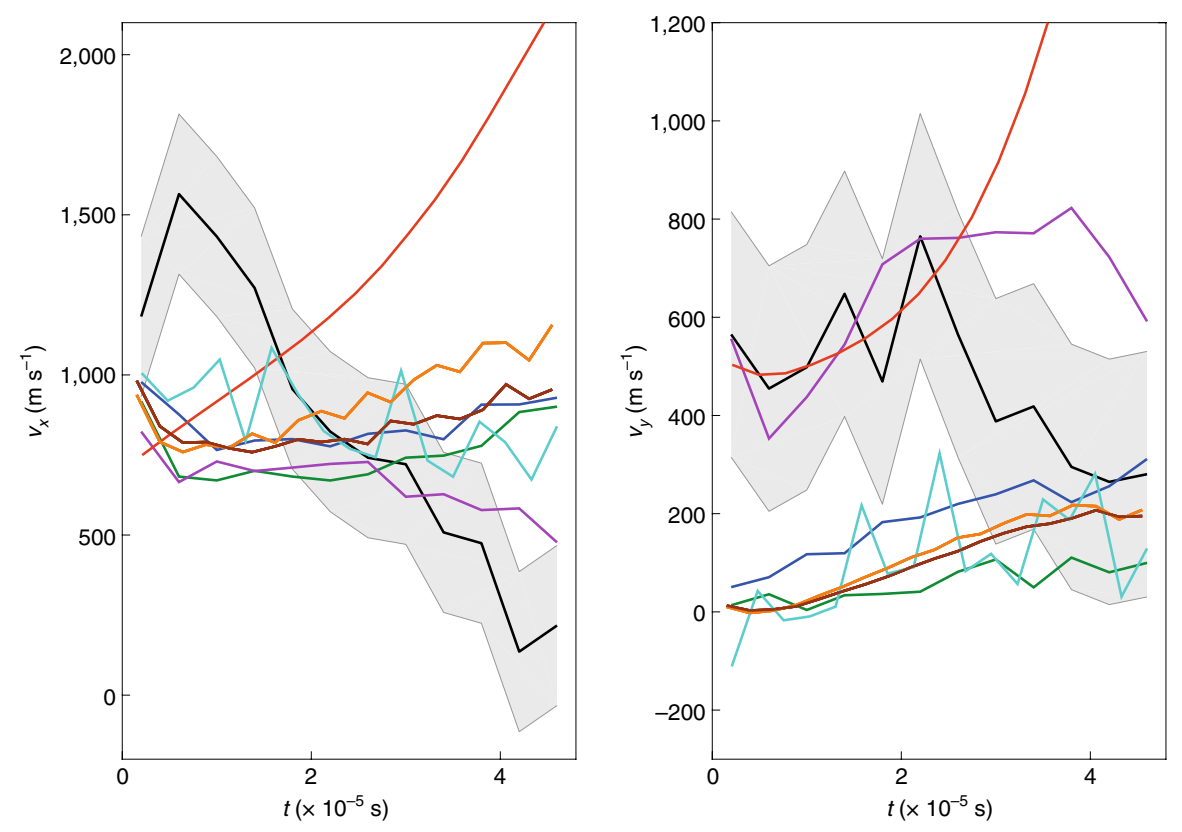

Fig. 3 | Numerical code validation. Validation of a number of numerical codes used to simulate the plasma periphery of fusion devices against the experimental measurement of the radial ( $v_{x i}$ left) and vertical ( $v_{y}$; right) velocity of a blob in TORPEX over time. The experimental data are plotted in black, together with the results from the simulations obtained from the codes BOUT++3D (dark blue) ${ }^{34}$, BOUT++2D (green) ${ }^{34}, \mathrm{GBS}$ (violet) ${ }^{30}$, GRILLIX (orange) ${ }^{33}$, GRILLIX with the Boussinesq approximation (brown) $)^{33}$, HESEL (red) ${ }^{35}$ and TOKAM $3 X$ (light blue) ${ }^{36}$. The grey shaded region represents the experimental uncertainty due to the finite spatial resolution of the probes. Data are from refs. ${ }^{32,33}$.

dynamics of blobs have been elucidated, blob motion has been actively controlled in TORPEX using electrostatically biased plates $^{20}$. Universal features in the statistical description of plasma fluctuations associated with blobs ${ }^{21}$ reveal that the mechanisms regulating the dynamics of turbulence and blobs can be generalized across devices and plasma realizations. A compendium of these results is shown in Fig. 2.

Fusion reactors will be operated in the burning plasma regime, in which the self-heating provided by the $\alpha$-particles - the by-products of the deuteriumtritium fusion reactions - dominates over the externally provided heating. These $\alpha$-particles are strongly suprathermal as they are generated at $3.5 \mathrm{MeV}$ in a quasiMaxwellian background plasma that has temperatures of the order of tens of $\mathrm{keV}$. Suprathermal ion dynamics, and in particular their cross-field transport, might be influenced by turbulent structures. Investigations of the interaction between suprathermal ions and turbulence using a miniaturized suprathermal $\mathrm{Li}^{6+}$ ion source and an electrostatic detector system, shown in Fig. 1, were performed.

By combining experimental data with fully validated numerical simulations, different regimes for the suprathermal ion transport were identified, which depend on the ion energy and turbulent eddy size and amplitude ${ }^{22,23}$. After a brief ballistic phase, in which the suprathermal ions do not interact significantly with the turbulence, the interaction causes the spreading of the entire suprathermal ion spectrum over superdiffusive, diffusive or sub-diffusive regimes. These non-classical transport properties are produced by the intermittent character of turbulence. The transition between the different non-diffusive regimes depends on both the normalized amplitude fluctuations and the injection energy normalized to the electron temperature, which determine the relative size of the turbulent structures and of the ion orbits. This verifies our understanding of the effect of orbit averaging of turbulence, that is, the fact that large and fast excursions in suprathermal ion trajectories across the positive and negative electrical potential structures of turbulence reduce the influence of turbulence on suprathermal ion transport. Good news for ITER and large burning plasma devices in general.

After reaching a basic understanding of the dynamics of turbulence and related structures in plasma configurations and conditions that are similar to those found in tokamaks, the focus shifted to the effect that these structures have on the propagation of radio-frequency waves in the plasma edge. Such externally launched microwaves will be used in ITER to control internal plasma instabilities, such as neoclassical tearing modes $^{24}$. The stabilization of such modes is crucial, as these can cause significant degradation of the plasma confinement and trigger plasma disruptions. The path of the microwave beams, which have wavelengths in the millimetre range as their frequency is of the order of the electron cyclotron (EC) frequency (of the order of $100 \mathrm{GHz}$ for ITER), must necessarily pass through regions where the tokamak plasma develops strong turbulence and blobs - similarly to what was observed in the TORPEX device. The scattering of the microwave beams must be quantified to understand and predict its effect on the core plasma stability. In more practical terms, the scattering also affects the specifications of the system for the generation and injection of microwaves, and the optimal approach to control the instabilities in real time, and hence the plasma fusion performance. Numerical and analytical studies suggest that in ITER, scattering could broaden EC beams by up to a factor of two. This would lead to a reduced stabilization of the plasma instabilities and may require modulation of the beam to recover the required performance ${ }^{25,26}$.

In TORPEX, the first direct experimental measurements of the scattering of millimetre-wave beams on plasma blobs were carried out with a dedicated millimetre-wave injection and detection system. A first-principles full-wave model predicts fluctuations of the millimetrewave power that are in agreement with experiments. These results represent a first important verification of the numerical codes used by the fusion community to simulate the propagation of EC beams in fusion devices ${ }^{27}$. Consequently, a similar set-up for the measurement of the scattering of MW-level millimetre-wave beams in the Tokamak of Variable Configuration $(\mathrm{TCV})^{28}$ at ITER relevant power levels and frequencies was developed. By using the paradigm obtained from the TORPEX experimental analysis and the relevant validated codes, similar physics features could be inferred despite the fact that considerably less information on the turbulent structures is available in TCV.

This example illustrates the importance of validating numerical codes to assess the maturity of the understanding of plasma dynamics and the predictive capabilities of simulations, which are a necessary tool to address the complexity of the numerical models needed for a realistic description of fusion devices ${ }^{29}$. As has been demonstrated with TORPEX, basic 
plasma devices can provide a very effective starting point for simulations of fusion devices. The simulations carried out for TORPEX constituted in fact the first step in the development of GBS, a state-of-the-art numerical code used today to simulate the plasma dynamics at the edge of fusion devices ${ }^{30}$.

Besides providing an ideal validation test bed for GBS, TORPEX has also served in validations of other edge simulation codes by performing a quantitative assessment of the agreement between the various numerical simulations and experimental data. The detailed diagnostic capabilities of TORPEX and the availability of global simulations of the device, enabled by its relatively simple magnetic configuration, led to the development of a rigorous validation methodology $y^{31}$, which is now routinely used for more complex tokamak conditions. A joint validation effort by four European groups involving five different $\operatorname{codes}^{32,33}$, which are based on different models and hypotheses, was carried out. The comparison between TORPEX measurements and simulations was based on a very large number of quantities, considering the uncertainties affecting them and the assumptions that are behind their evaluation. Figure 3 illustrates a comparison of the TORPEX results with different codes, focusing on the propagation velocity of blobs in the direction perpendicular to the magnetic field. This comparison allowed to disentangle the basic physics elements behind the motion of blobs.
Although the ITER design is now essentially frozen, its optimal operation would benefit from addressing some basic plasma physics questions in simplified configurations, which could be specifically designed. For the step after ITER, even the conceptual design can still be influenced by fundamental discoveries. Among the possible examples of the open physics questions are the dynamics of runaway electrons, which are subject to a progressively decreasing collisional drag while being accelerated, resulting from abrupt terminations of plasma discharges. Other open issues include the effect of different magnetic topologies on the exhaust of plasma particles and heat at the edge, as well as the mechanisms for locally inducing electrical currents in the plasma using waves. Basic plasma research naturally also constitutes a pole of attraction for the interest of young scientists, and a platform for the development of skills and novel ideas, even in academic environments that are not prone to big science investments. These are fundamental assets for the transgenerational challenge that the development of fusion energy embodies.

\section{Ambrogio Fasoli (iD*, Ivo Furno and Paolo Ricci \\ Ecole Polytechnique Fédérale de Lausanne (EPFL), Swiss Plasma Center (SPC), Lausanne, Switzerland. *e-mail:ambrogio.fasoli@epfl.ch}

Published online: 5 August 2019 https://doi.org/10.1038/s41567-019-0622-5
References

1. ITER Physics Basis Editors et al. Nucl. Fusion 39, 2137-2174 (1999).

2. Zohm, H. et al. Nucl. Fusion 53, 073019 (2013).

3. Stangeby, P. C. The Plasma Boundary of Magnetic Fusion Devices (Institute of Physics Publishing, 2000).

4. Fasoli, A. et al. Nucl. Fusion 47, S264-S284 (2007).

5. Experiments at the Frontier of Fundamental Plasma Physics Special issue of the J. Plasma Phys. Vol. 81 (2015).

6. Yamada, M., Kulsrud, R. \& Ji, H. Rev. Mod. Phys. 82 , 603-664 (2010).

7. Pierre, T., Leclert, G. \& Braun, F. Rev. Sci. Instrum. 58, 6-11 (1987).

8. Matsukuma, M. et al. Phys. Lett. A 314, 163-167 (2003).

9. Bohlin, H. et al. Rev. Sci. Instrum. 85, 023501 (2014).

10. Krause, N., Lechte, C., Stöber, J. \& Stroth, U. Rev. Sci. Instrum. 73, 3474-3481 (2002).

11. Tynan, G. et al. Phys. Plasmas 11, 5195-5203 (2004)

12. Fattorini, L. et al. Plasma Phys. Control. Fusion 54, 085017 (2012).

13. Trasarti-Battistoni, R., Draghi, D., Riccardi, C. \& Roman, H. Phys. Plasmas 9, 3369-3377 (2002).

14. Fasoli, A. et al. Plasma Phys. Control. Fusion 52, 124020 (2010).

15. Furno, I. et al. J. Plasma Phys. 81, 345810301 (2015).

16. Zweben, S. J. Phys. Fluids 28, 974-982 (1985).

17. Furno, I. et al. Phys. Rev. Lett. 100, 055004 (2008).

18. Theiler, C. et al. Phys. Rev. Lett. 103, 065001 (2009).

19. Avino, F. et al. Phys. Rev. Lett. 116, 105001 (2016).

20. Theiler, C. et al. Phys. Rev. Lett. 108, 065005 (2012).

21. Labit, B. et al. Phys. Rev. Lett. 98, 255002 (2007).

22. Gustafson, K. et al. Phys. Rev. Lett. 108, 035006 (2012).

23. Bovet, A. et al. Phys. Rev. Lett. 113, 225001 (2014).

24. Gantenbein, G. et al. Phys. Rev. Lett. 85, 1242-1245 (2000).

25. Poli, E. et al. Nucl. Fusion 55, 013023 (2015).

26. Sysoeva, E. V. et al. Nucl. Fusion 55, 033016 (2015).

27. Chellai, O. et al. Phys. Rev. Lett. 120, 105001 (2018).

28. Chellaï, O. et al. Plasma Phys. Control. Fusion 61, 014001 (2018)

29. Fasoli, A. et al. Nat. Phys. 12, 411-423 (2016).

30. Ricci, P. et al. Plasma Phys. Control. Fusion 54, 124047 (2012).

1. Ricci, P. et al. Phys. Plasmas 18, 032109 (2011).

32. Riva, F. et al. Plasma Phys. Control. Fusion 58, 044005 (2016).

33. Stegmeir, A. et al. Plasma Phys. Control. Fusion 60, 035005 (2018).

34. Dudson, B. D. et al. Comput. Phys. Commun. 180 1467-1480 (2009).

35. Nielsen, A. H. et al. Phys. Lett. A 379, 3097-3101 (2015). 36. Tamain, P. et al. Contrib. Plasma Phys. 54, 555-559 (2014). 